\title{
Distance perception and the ambiguity of visual stimulation: A theoretical note
}

\author{
E. STURE ERIKSSON* \\ University of Uppsala, Lippsala, Sweden
}

\begin{abstract}
Certain current views postulate that visual perception, especially of distance, is a function of optical stimulation alone. It is shown here that the optical array does not, in fact, specify absolute distance unambiguously, for either a stationary or a moving $O$. In view of this ambiguity of optical information, a more complex theory, comprising both visual and nonvisual information, is needed to explain veridical perception.
\end{abstract}

Certain current views assume that there is a direct relation between visual perception and optical stimulation, and that nonoptical stimuli and processes need not be considered in a theory of visual perception. For example, it has been hypothesized (Gibson, 1959) that "...for every aspect or property of the phenomenal world of an individual in contact with his environment, however subtle, there is a variable of the energy flux at his receptors, however complex, with which the phenomenal property would correspond if a psychophysical experiment could be performed [p. 465]... The stimulus variables for vision must exclusively be found in a textured optical array, supplemented by the transformation relating a simultaneous pair of them, and by the transformations relating a continuous sequence of momentary arrays [p. 474]."

If the hypothesis of an unambiguous correspondence between optical variables and visual perception were true, a simple nativist theory of space perception would be tenable and attractive. It would be sufficient to assume that the organism was endowed with a visual receptor system tuned to the unambiguous information. But if it is possible to show that the optical information is ambiguous, it will be necessary to postulate a system or a mechanism which reduces the ambiguity and yields veridical perception.

Typically, we perceive objects as located at a definite distance and having a definite size. Can purely optical variables be found that uniquely specify an object's absolute distance and size? The transactionalists pointed out that any pattern of retinal stimulation is fundamentally ambiguous. in that any one of an infinite number of physical arrangements could have produced that retinal image (see, e.g.. Ittelson, 1960). It has often been assumed, however, that the ambiguity can be resolved if texture gradients and the optical changes produced by an O's movements are taken into account.

The purpose of this note is to prove that optical stimulation alone is always an ambiguous indicant of

*The author is indebted to C. S. Harris for valuable comments on this paper. absolute distance and size, even in a textured field. The ambiguity is present not only for a stationary $\mathrm{O}$, but also for an $\mathrm{O}$ who moves freely in any direction. However, since recent research has indicated that accommodation and accommodative convergence may have some effects on distance perception in near space (Gogel, 1961; Wallach \& Norris, 1963; Richards \& Miller, 1969), the present analysis is valid for distance perceptions unaffected by the above-mentioned factors.

(1) Is absolute distance unambiguously specified by a static texture gradient? Two classical formulations of the problem of distance perception (Gibson, 1950, p. 62). as well as the geometrical basis for the present analysis, are shown in Fig. 1. Consider a monocular, stationary $\mathrm{O}$ and a surface with uniform texture. As Fig. 1 shows, the proximal stimulation generated by Surface 1 is identical to the proximal stimulation generated by a second surface and an infinity of surfaces with uniform textures but lying at different distances from the eye and having elements of correspondingly different sizes. Thus. veridical distance perception of the distance of the surface and of objects resting on it cannot be deduced from the retinal texture gradient. The dilemma cannot be solved by arguing that there is a psychological size scale that assigns a definite range of subjective sizes to a

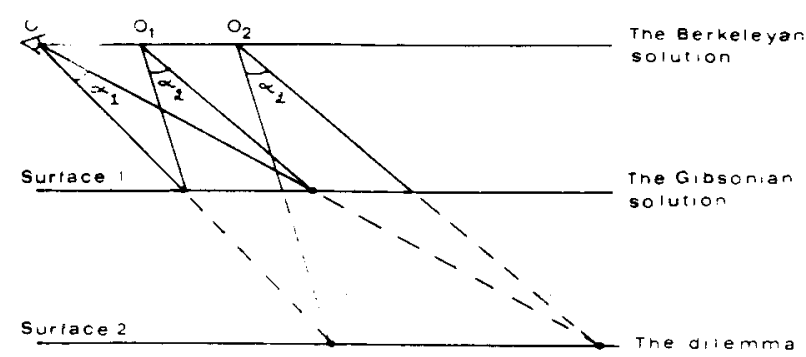

Fig. 1. Three formulations of the problem of distance perception: (a) The classical solution proposed by Berkeley according to which distance is not directly perceived. (b) The gradient theory which reduces distance perception to the perception of distances along a surface. (c) The theoretical dilemma arising when the existence of different surfaces at different distances from the eye is postulated. 


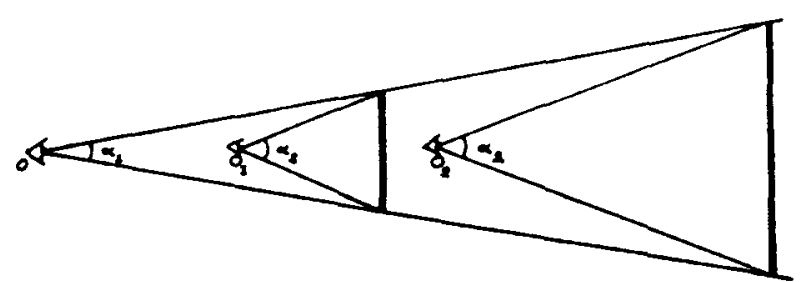

Fig. 2. The ambiguity of size-distance information for two Os $\left(\mathrm{O}_{1}\right.$ and $\left.\mathrm{O}_{2}\right)$ moving from the point 0 toward different rigid distal objects with differences in the registration of their own motions.

given retinal gradient (Gibson, 1950, pp. 180-181). A fixed size scale cannot explain veridical perception of surfaces at different distances and with different element sizes. The difficulty cannot be overcome by postulating scales of "higher order," because the critical ambiguity concerns the basic unit size underlying all possible relations and scales. If different assumed sizes or scales are postulated for particular proximal stimulus texture, it would be equivalent to the classical empiristic interpretation (Ittelson, 1960).

(2) Is perceived distance unambiguously specified by a motion parallel to the ground? If the $O$ in Fig. 1 moves from 0 to $0_{1}$, the eye receives a changing stimulation, $\alpha_{1}-\alpha_{2}$. If the $O$ correctly registers the length of Path $0 \cdot 0_{1}$. he can perceive the surface as being at a certain distance from him (Surface 1). But if he overestimates his motion and perceives the motion path to be from 0 to $0_{2}$, the same proximal change from $\alpha_{1}$ to $\alpha_{2}$ will correspond to a surface at a larger distance from him (Surface 2). It follows that the visual change in itself is ambiguous with regard to distance in this situation.

(3) Does locomotion toward a surface or object exhibit the same kind of ambiguity? Even if the $O$ in Fig. 2 assumes that the object or surface is rigid and stationary, movement toward the object (from 0 to $0_{1}$ ) generates a proximal pattern of expansion (from $\alpha_{1}$ to $\alpha_{2}$ ). which is identical to the expansion pattern for a locomotion with a larger velocity toward an object at a larger distance from the eye (from 0 to $\mathrm{O}_{2}$ ). A correctly perceived distance cannot be explained on the sole basis of the proximal pattern of expansion, the $O$ must also correctly register his own velocity, displacement, or initial position.

Could the $\mathrm{O}$ use the optical information to correctly register his movement (Gibson, 1950, pp. 124, 224; 1966, p. 200)? Since such "visual kinesthesis" must be based on information via the retina and since, in our example, that information would be identical for movements from 0 to $0_{1}$ and from 0 to $0_{2}$, visual kinesthesis must also give ambiguous information. Consequently, to achieve veridical perception, the registration of the $O$ 's own motion state must be derived from kinesthetic. vestibular, or cognitive systems. i.e.. some kind of nonvisual information.

(4) Is the ambiguity absent in more complex environments with more complex movements? In a recent book, it is suggested that a moving $O$ generates a motion perspective which is unambiguous: "A careful examination of the two arrays in Figure 10.6 [showing how the optic array is transformed when a seated $O$ stands up in a room] ... will confirm the hypothesis that the optical flow from one to the other, the transformation. is specific to that layout of surfaces and to no other. There could be a family of optically equivalent rooms for the seated man; there could be another family of optically equivalent rooms for the standing man; but there could only be that particular rectangular room for that particular motion perspective obtained when he stands up [Gibson, 1966, p. 199]."

Figure 3 shows that even this complex O-produced optical transformation really is ambiguous. Imagine two identical twins, $\mathrm{O}_{1}$ and $\mathrm{O}_{2}$, moving as in the above example. The only difference between the twins is assumed to be that $\mathrm{O}_{1}$ registers his own motion correctly while $\mathrm{O}_{2}$ overestimates it. If the twins move in the same physical path, $0-0_{1}$, it follows that $O_{1}$ will perceive the room veridically, while $\mathrm{O}_{2}$ (who perceives his own motion to be $0-0_{2}$ ) will perceive a room which is correspondingly larger.

Thus, one and the same optical flow pattern may correspond to quite different distal room sizes, depending on the extent of the 0 's motion $\left(0-0_{1}\right.$ vs $0-0_{2}$ ). However, information about the absolute extent of that motion is not given by the optical flow (see Point 3 above). Hence, a given optical flow could be produced by different magnitudes of $\mathrm{O}$ motion, and consequently by rooms of different sizes.

Since the above arguments demonstrate that the optical flow in itself is ambiguous, it follows that it is not possible to explain veridical perception without

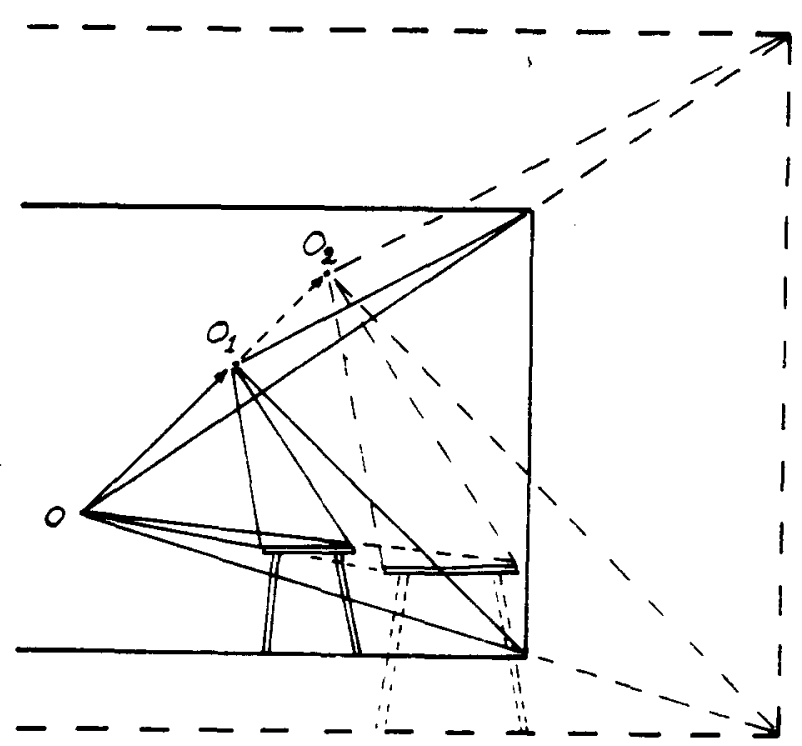

Fig. 3. The ambiguity of size-distance information for two $O s$ moving in a rectangular room. 
assuming that the $\mathrm{O}$ correctly registers his own motion. Since veridical perception is obtained in a great variety of normal situations, it must be that the O's own motion typically is correctly registered. From a theoretical point of view. however. it makes a great difference whether veridicality is guaranteed by unambiguous optical information or requires an interaction between ambiguous optical information and nonvisual information.

In summary, it is concluded that monocular visual stimulation is characterized by a basic ambiguity, an ambiguity which holds for both a stationary and a moving $O$. Therefore, for the stationary $O$, an explanation of veridical distance and size perception based exclusively on monocular optical information is untenable. ${ }^{1}$ An adequate explanation must either take empiristic factors into account or else postulate that other kinds of nonvisual information is sufficient. e.g., an innate registration of body height. ${ }^{2}$ With regard to veridical size and distance perception under motion conditions, a nativistic explanation at least requires an assumption concerning registration of the O's own motion, based on kinesthetic or vestibular information. The alternative is to assume that ambiguity is resolved by way of stored information in the empiristic sense. In any case. it must be concluded that a general theory of space perception cannot be based solely on optical information but has to consider nonvisual information as well.

\section{REFERENCES}

Epstein, W., \& Park, J. Examination of Gibson's psychophysical hypothesis. Psychological Bulletin, 1964. 61. 180-196.

Flock, H. A possible optical basis for monocular slant perception. Psychological Review, 1964, 71, 380-391.

Gibson, J. J. The perception of the visual world. Boston: Houghton Mifflin. 1950.

Gibson. J. J. Perception as a function of stimulation. In S. Koch (Ed.), Psychology: A study of a science. Vol. 1. New York: McGraw-Hill. 1959. Pp. 456-501.

Gibson. J. J. The senses considered as perceptual systems. Boston: Houghton Mifflin. 1966.

Gibson. J. J., Olum, P.. Rosenblatt, F. Parallax and perspective during aircraft landings. American Journal of Psychology. $1955,68,372-375$.
Gibson, E., Gibson, J. J., Smith. O. W., \& Flock, H. Motion parallax as a determinant of perceived depth. Journal of Experimental Psychology, 1959, 58, 40-51.

Gogel, W. C. Convergence as a cue to absolute distance. Journal of Psychology, 1961, 52, 287-301.

Hay, J. C. Optical motions and space perception: An extension of Gibson's analysis. Psychological Review, 1966, 73, $550-565$.

Ittelson, W. H. Visual space perception. New York: Springer, 1960.

Richards. W., \& Miller, J. F. Convergence as a cue to depth. Perception \& Psychophysics, 1969, 5, 317-320.

Wallach, H., \& Norris, C. M. Accommodation as a distance cue. American Journal of Psychology, 1963, 76, 659-664.

\section{NOTES}

1. It should be noted that Gibson has almost never explicitly discussed absolute distance and size perception. The vast majority of his theoretical explanations may well apply to relative perceptions. However, because he so seldom reminds the reader that he is discussing relative perceptions when he refers simply to "distance" or "depth," and because he says that his theory applies to normal aspects of perception, there is a widespread assumption that the theory applies to absolute perceptions as well. Although several writers have, in passing, noted the ambiguity of optical stimulation that is the topic of this note, they have done so in ways that easily escape notice (see, e.g., Gibson, Olum, \& Rosenblatt, 1955; Gibson, Gibson, Smith, \& Flock, 1959; Epstein \& Park, 1964; Hay, 1966; Flock, 1964).

2. Gibson has suggested $(1959$, pp. 475,481$)$ that absolute distance perception may depend on the sight of the O's nose at the edge of the visual field: ". . the specific distance of any point on the surface would be given by the degree of density, disparity, and motility at that point in the array relative to the whole gradient. The distance from 'here to there,' in short, is probably produced by the difference with respect to these variables between the image of the nose in the field of view (the visual 'here') and the image of the object (which is 'there') [1959. p. 475]." Using the nose as a reference point, however, will not turn a relative distance perception into an absolute one, since the length and shape of the nose, and its distance from the ey'e, cannot be determined from purely optical information. Hence. all that could be determined is that the distant object is "so many times as far away as the visible part of my nose." The same reasoning applies to sight of any other part of the body.

(Received for publication June 28, 1972; revision received November 22, 1972.) 